\title{
A RAMSEY THEOREM FOR BIASED GRAPHS
}

\author{
PETER NELSON AND SOPHIA PARK
}

\begin{abstract}
A biased graph is a pair $(G, \mathcal{B})$, where $G$ is a graph and $\mathcal{B}$ is a collection of 'balanced' circuits of $G$ such that no $\Theta$ subgraph of $G$ contains precisely two balanced circuits. We prove a Ramsey-type theorem, showing that if $(G, \mathcal{B})$ is a biased graph which $G$ is a very large complete graph, then $G$ contains a large complete subgraph $H$ such that the set of balanced cycles within $H$ has one of three specific, highly symmetric structures, all of which can be described naturally via group-labellings.
\end{abstract}

\section{INTRODUCTION}

A $\Theta$-subgraph of a graph $G$ is a connected subgraph of $G$ whose edge set is the union of three edge-disjoint $x y$-paths for some pair of distinct vertices $x$ and $y$. Such a subgraph contains exactly three circuits of $G$. A biased graph is a pair $(G, \mathcal{B})$ where $G$ is a graph and $\mathcal{B}$ is a collection of circuits of $G$, called the 'balanced' circuits, so that no $\Theta$-subgraph of $G$ contains precisely two circuits in $\mathcal{B}$.

As was shown in Zaslavsky's work 2 defining these objects, a natural class of biased graphs arises by assigning orientations and labels from a group to the edges of a graph $G$, and indeed, biased graphs arose as an abstraction of these 'group-labelled graphs'. We explain the construction briefly here. For a group $\Gamma$, a $\Gamma$-labelled graph is a pair $(\vec{G}, \gamma)$, where $\vec{G}$ is an orientation of an undirected graph $G=(V, E)$, (that is, $G$ together with an assignment of a 'head' in $\{u, v\}$ to each undirected edge $u v \in E$, towards which the edge is 'oriented') and $\gamma: E \rightarrow \Gamma$ is a function.

For each circuit $C$ of $G$ with vertices $v_{1}, \ldots, v_{k}$ and edges $e_{1}, \ldots, e_{k}$ where $v_{i}, v_{i+1}$ are the ends of $e_{i}$ and $v_{k+1}=v_{1}$, let $\pi(C)=\prod_{i=1}^{k} \gamma^{\prime}\left(e_{i}\right)$, where $\gamma^{\prime}\left(e_{i}\right)=\gamma\left(e_{i}\right)$ if $e_{i}$ is oriented from $v_{i}$ to $v_{i+1}$, and $\gamma^{\prime}\left(e_{i}\right)=\gamma\left(e_{i}\right)^{-1}$ otherwise. We say that $C$ is balanced if $\pi(C)$ is the identity in $\Gamma$; although $\pi(C)$ may depend on the particular choice of indexing of the vertices in $C$, associativity implies that the definition of balanced does not, even if $\Gamma$ is nonabelian. Zaslavsky showed that $(G, \mathcal{B})$ is always a biased graph for the set $\mathcal{B}$ of circuits of $G$ that are balanced in the above sense. It is known, however, that not all biased graphs arise 
from group-labelled graphs in this way; in fact, [1 shows that there exist infinite antichains (with respect to biased subgraph containment) of biased (multi-)graphs on three vertices that do not.

A biased subgraph of $(G, \mathcal{B})$ is a pair $\left(H, \mathcal{B}^{\prime}\right)$ where $H$ is a subgraph of $G$, and $\mathcal{B}^{\prime}$ is the intersection of $\mathcal{B}$ with the set of circuits of $H$. We prove the following Ramsey-type theorem for biased graphs; $K_{n}$ denotes the complete graph with vertex set $[n]=\{1, \ldots, n\}$.

Theorem 1.1. For every integer $s \geq 1$, there exists an integer $n$ such that, if $(G, \mathcal{B})$ is a biased graph with $G \cong K_{n}$, then $(G, \mathcal{B})$ has a biased subgraph $\left(H, \mathcal{B}^{\prime}\right)$ with $H \cong K_{s}$ that arises from a $\Gamma$-labelled graph for some finite cyclic group $\Gamma$.

In fact, we refine the conclusion to show that $\left(H, \mathcal{B}^{\prime}\right)$ arises from a graph with one of three very specific types of group-labelling, which we now define.

Let $\vec{K}_{n}$ be the orientation of $K_{n}=([n], E)$, where every edge with ends $i<j$ is oriented from $i$ to $j$. Let $m=\left(\begin{array}{l}n \\ 2\end{array}\right)$ and $\Gamma$ be a cyclic group of order at least $2^{m}$ with generator $g$. Let $S \subseteq \Gamma$ be a set of size $m$ such that no two distinct subsets of $S$ have the same product; such a set $S$ exists, as we can take $S=\left\{g^{2^{i}}: 0 \leq i<m\right\}$. Let $\gamma^{\mathbf{u}}: E \rightarrow \Gamma$ be a labelling assigning distinct elements of $S$ to each edge. Let $\gamma^{\mathbf{o}}: E \rightarrow \Gamma$ be a labelling such that every edge receives a label in $S$, and two edges receive the same label if and only if they have the same head (that is, larger end).

For each integer $a \geq 0$, let $\Gamma$ be a multiplicative cyclic group that is infinite if $a=0$ and has size $a$ otherwise, and let $g$ be a generator for $\Gamma$. Let $\gamma^{a}: E \rightarrow \Gamma$ assign a label of $g$ to every edge.

Let $K_{n}^{\mathbf{u}}, K_{n}^{\mathbf{o}}, K_{n}^{a}$ be the biased graphs of the group-labelled graphs corresponding to $\vec{K}_{n}$ and the labellings $\gamma^{\mathbf{u}}, \gamma^{\mathbf{o}}$ and $\gamma^{a}$ (for $a \geq 0$ ) respectively. While the first two ostensibly depend on the choice of $\Gamma$ and $S$, Lemma 2.1 will show that in fact they do not. We can now state a refinement of our main theorem.

Theorem 1.2. For every integer $s \geq 1$, there exists an integer $n$ such that, if $(G, \mathcal{B})$ is a biased graph with $G \cong K_{n}$, then $(G, \mathcal{B})$ has a biased subgraph isomorphic to either $K_{s}^{\mathbf{u}}, K_{s}^{\mathbf{o}}$, or $K_{s}^{a}$ for some $a>0$.

In all three cases, there are simple combinatorial descriptions for the set of balanced cycles in the subgraph. We state these here, and will prove them in Lemma 2.1. For a $k$-circuit $C$ of $K_{n}$, let $v_{1}, \ldots, v_{k}$ be the unique ordering of $V(C)$ for which $E(C)=\left\{v_{1} v_{2}, \ldots, v_{k-1} v_{k}, v_{k} v_{1}\right\}$, and $v_{1}=\min (C)$ while $v_{2}<v_{k}$. Set $v_{0}=v_{k}$. For $i \in[k]$, we say that 
the edge $v_{i-1} v_{i}$ is positive in $C$ if $v_{i}>v_{i-1}$, and negative otherwise. We will show that

- $K_{s}^{\mathbf{u}}$ has no balanced circuits,

- a circuit $C$ is balanced in $K_{s}^{\mathbf{o}}$ if and only if the edges of $C$ alternate between positive and negative, and

- a circuit $C$ is balanced in $K_{s}^{a}$ if and only if $a \mid d$, where $d$ is the difference between the number of positive edges in $C$ and the number of negative edges in $C$.

Note that if $a=1$ in the third case, then every circuit is balanced, and if $a=2$ then precisely the even circuits are balanced; these natural cases are therefore both possible outcomes of Theorem 1.2 .

The function $n=n(s)$ implicitly defined in Theorem 1.2 depends on Ramsey numbers for 4-colouring the edges of a complete 4-uniform hypergraph, and therefore corresponds to a tower of exponents of constant height. One could shorten our proof in exchange for much worse numbers (invoking Ramsey's theorem for $f(s)$-uniform hypergraphs with $g(s)$ colours, where $f$ and $g$ are respectively linear and exponential in $s)$. We also prove a bipartite version of our main theorem. Here the outcomes simplify and our bound is small enough to include explicitly.

Theorem 1.3. Let $t \geq 1$. If $n \geq 2^{2^{2^{4 t}}}$ and $(G, \mathcal{B})$ is a biased graph with $G \cong K_{n, n}$, then $(G, \mathcal{B})$ has a biased subgraph $\left(H, \mathcal{B}^{\prime}\right)$ with $H \cong K_{t, t}$ such that $\mathcal{B}^{\prime}$ is either empty, or the set of all circuits in $H$.

As part of the work we do to get our better bound in Theorem [1.2, we obtain a result of independent interest, Lemma 5.2, that gives a nearly complete characterisation of any biased complete graph in which only certain 4-circuits are required to be balanced. We state a slightly simplified version here. For a biased graph $(G, \mathcal{B})$ and a vertex $v$ of $G$, we write $(G, \mathcal{B})-v$ for the biased subgraph $\left(H, \mathcal{B}^{\prime}\right)$ with $H=G-v$.

Theorem 1.4. Let $n \geq 5$ and $(G, \mathcal{B})$ be a biased graph with $G=K_{n}$. If, for all $v_{1}, v_{2}, v_{3}, v_{4} \in V(G)$ for which either $v_{1}<v_{2}<v_{4}<v_{3}$ or $v_{1}<v_{3}<v_{2}<v_{4}$, the circuit with edge set $\left\{v_{1} v_{2}, v_{2} v_{3}, v_{3} v_{4}, v_{4} v_{1}\right\}$ is balanced, then $(G, \mathcal{B})-n=K_{n-1}^{a}$ for some $a>0$.

The single vertex deletion turns out to be necessary; in fact, Theorem 5.4 will show that if the statement is strengthened to assert that $(G, \mathcal{B})$ itself has the form $K_{n}^{a}$, then there are doubly exponentially many counterexamples.

\section{ORDERED BIASED GRAPHS}

Our biased graphs hereon are always complete. While we could phrase all our results in terms of graphs $K_{n}$ with vertex set $\{1, \ldots, n\}$, 
when passing to subgraphs it is more convenient to state them in terms of biased graphs with a fixed linear ordering on the vertex set; this will also allow us to state a slightly stronger, 'ordered' version of our main theorem. An ordered graph is a pair $(G, \leq)$ where $G$ is a graph and $\leq$ is a total ordering on $V(G)$, and an ordered biased graph is a triple $(G, \mathcal{B}, \leq)$, where $(G, \mathcal{B})$ is a biased graph and $(G, \leq)$ is an ordered graph. Two ordered biased graphs $\left(G_{1}, \mathcal{B}_{1}, \leq_{1}\right)$ and $\left(G_{2}, \mathcal{B}_{2}, \leq_{2}\right)$ are isomorphic if there is a graph isomorphism from $G_{1}$ to $G_{2}$ that preserves both the property of being balanced for a circuit, and the ordering of the vertices. We defined our special biased graphs $K^{\mathbf{u}}(n), K^{\mathbf{o}}(n)$ and $K^{a}(n)$ to each have a fixed vertex set $[n]$; from here on we view them as ordered biased graphs with the usual ordering on $[n]$.

We write either $\left[v_{1}, \ldots, v_{k}\right]$, or $\left[v_{i}: i \in \mathbb{Z}_{k}\right]$ for the circuit with edge set $\left\{v_{k} v_{1}, v_{1} v_{2}, \ldots, v_{k-1} v_{k}\right\}$. Each $k$-circuit has $2 k$ such representations. Given a circuit $C$ in an ordered graph $(G, \leq)$, define the canonical ordering of $C$ to be the unique sequence $v_{1}, \ldots, v_{k}$ for which $C=\left[v_{1}, \ldots, v_{k}\right]$ and $v_{1}=\min (V(C))$ while $v_{2}<v_{k}$; this distinguished ordering was considered but not named in the introduction. We say that two $k$-circuits $C, C^{\prime}$ are similar in $(G, \leq)$ if their canonical orderings $v_{1}, \ldots, v_{k}$ and $v_{1}^{\prime}, \ldots, v_{k}^{\prime}$ are such that $v_{i}<v_{j}$ if and only if $v_{i}^{\prime}<v_{j}^{\prime}$. This is an equivalence relation on the set of all $k$-circuits of $G$, and for each $k$-element subset $X$ of $G$, each equivalence class contains precisely one circuit with vertex set $X$.

We will be especially concerned with two special similarity classes of 4-circuits. Call a 4-circuit $C$ a 1324-circuit if its canonical ordering $v_{1}, v_{2}, v_{3}, v_{4}$ satisfies $v_{1}<v_{3}<v_{2}<v_{4}$ and define a 1243-circuit similarly. Note that if $V=[n]$ with $n \geq 4$, then $[1,3,2,4]$ and $[1,2,4,3]$ are natural examples of these two types of circuit.

A circuit $\left[v_{i}: i \in \mathbb{Z}_{k}\right]$ is oscillating if for each $i \in \mathbb{Z}_{k}$, the vertex $v_{i}$ satisfies either $v_{i}<\min \left(v_{i-1}, v_{i+1}\right)$ or $v_{i}>\max \left(v_{i-1}, v_{i+1}\right)$. This clearly does not depend on the choice of indexing, and is equivalent to the definition earlier that the edges of $C$ alternate between 'positive' and 'negative'. Oscillating 4-circuits are precisely the 1243-circuits.

For each circuit $C=\left[v_{i}: i \in \mathbb{Z}_{k}\right]$, let $\delta(C)=\left|\delta_{0}(C)\right|$, where

$$
\delta_{0}(C)=\left|\left\{i \in \mathbb{Z}_{k}: v_{i}>v_{i-1}\right\}\right|-\left|\left\{i \in \mathbb{Z}_{k}: v_{i}<v_{i-1}\right\}\right| .
$$

Cyclically permuting the $v_{i}$ does not change either term in $\delta_{0}$, while reversing the order of the $v_{i}$ swaps the two terms, changing only the sign of $\delta_{0}$; thus, the parameter $\delta(C)$ is a well-defined function of $C$ itself, not on the given indexing of its vertices. Fixing the ordering of $V(C)$ to be the canonical one, we see that the value of $\delta$ is the absolute difference between the number of 'positive' and 'negative' edges in $C$ as defined 
in the introduction. Oscillating circuits clearly satisfy $\delta=0$, since the two sets defining $\delta_{0}$ have equal size. Note that $\delta(C) \leq|V(C)|-2$ and that $\delta(C)$ and $|V(C)|$ have the same parity.

Given these definitions, we can now prove the combinatorial characterisations of the special biased graphs $K_{n}^{\mathbf{u}}, K_{n}^{\mathbf{o}}$ and $K_{n}^{a}$ that were stated earlier. Recall that they all arise from group-labellings of the orientation $\vec{K}_{n}$ of $K_{n}$ in which edges are oriented 'upwards'; the definitions of the relevant labellings are recalled in the proof below.

Lemma 2.1. If $n \geq 1$ is an integer, then

(i) The set of balanced circuits of $K^{\mathbf{u}}(n)$ is empty,

(ii) The balanced circuits of $K^{\mathbf{o}}(n)$ are precisely the oscillating circuits of $\left(K_{n}, \leq\right)$, and

(iii) if $a \geq 0$ is an integer, then the balanced circuits of $K^{a}(n)$ are precisely the circuits $C$ of $\left(K_{n}, \leq\right)$ for which a $\mid \delta(C)$.

Proof. Let $G=(V, E) \cong K_{n}$. Let $\Gamma$ be a cyclic group and $S \subseteq \Gamma$ be an $\left(\begin{array}{l}n \\ 2\end{array}\right)$-element set whose subsets all have distinct products.

Let $\gamma=\gamma^{\mathbf{u}}: E \rightarrow \Gamma$ be a function assigning distinct elements of $S$ to each edge, so $K_{n}^{\mathbf{u}}$ arises from $\left(\vec{K}_{n}, \gamma^{\mathbf{u}}\right)$. . Let $C=\left[v_{1}, \ldots, v_{k}\right]$ be a cycle of $K_{n}$. Then $\pi(C)=\prod_{i \in \mathbb{Z}_{k}}\left(\gamma^{\mathbf{u}}\left(v_{i} v_{i+1}\right)\right)^{-b_{i}}$ where each $b_{i}$ is -1 or 1 . So $\pi(C)$ is the identity if and only if $\prod_{i: b_{i}=1} \gamma^{\mathbf{u}}\left(v_{i} v_{i+1}\right)=$ $\prod_{i: b_{i}=-1} \gamma^{\mathbf{u}}\left(v_{i} v_{i+1}\right)$. The two sides of this equality are products of distinct subsets of $S$, so this cannot occur; thus, all circuits of $K^{\mathbf{u}}(n)$ are unbalanced, as required.

Now let $\gamma^{\mathbf{o}}: E \rightarrow \Gamma$ be a function assigning an element of $S$ to each edge, such that two edges receive the same label if and only if their larger end (head) is the same, so $K_{n}^{\mathbf{o}}$ arises from $\left(\vec{K}_{n}, \gamma^{\mathbf{o}}\right)$. For each $v \in[n]$, let $s_{v} \in S$ be the label assigned to the edges whose head is $v$. Let $C=\left[v_{i}: i \in \mathbb{Z}_{k}\right]$. Since each edge is oriented from its minimum to its maximum, we have

$$
\begin{aligned}
\pi(C) & =\prod_{i \in \mathbb{Z}_{k}: v_{i+1}>v_{i}} \gamma^{\mathbf{o}}\left(v_{i} v_{i+1}\right) \prod_{i \in \mathbb{Z}_{k}: v_{i+1}<v_{i}} \gamma^{\mathbf{o}}\left(v_{i} v_{i+1}\right)^{-1} \\
& =\left(\prod_{i \in \mathbb{Z}_{k}: v_{i+1}>v_{i}} \gamma_{i+1}\right)\left(\prod_{i \in \mathbb{Z}_{k}: v_{i+1}<v_{i}} \gamma_{i}\right)^{-1}
\end{aligned}
$$

Note that since $v_{1}, \ldots, v_{k}$ are distinct, each of the two terms is a product of distinct elements of $S$. Therefore, by the choice of $S$, the circuit $C$ is balanced if and only if $\left\{i \in \mathbb{Z}_{k}: v_{i+1}>v_{i}\right\}=\left\{i \in \mathbb{Z}_{k}: v_{i+1}<v_{i}\right\}$. 
This occurs precisely when each $v_{i}$ either satisfies $v_{i}>\min \left(v_{i-1}, v_{i+1}\right)$ or $v_{i}>\max \left(v_{i-1}, v_{i+1}\right)$ : that is, when $C$ is oscillating. This gives (iii).

Finally, let $a \geq 0$ be an integer and let $\Gamma_{a}$ be a cyclic group with generator $g$ such that $\Gamma$ is infinite if $a=0$, and $|\Gamma|$ has order $a$ otherwise. Let $\gamma^{a}: E \rightarrow \Gamma_{a}$ be the labelling assigning $g$ to every edge, so $K_{n}^{a}$ arises from $\left(\vec{K}_{n}, \gamma^{a}\right)$. Then, for a cycle $C=\left[v_{i}: i \in \mathbb{Z}_{k}\right]$, we have

$$
\begin{aligned}
\pi(C) & =\prod_{i \in \mathbb{Z}_{k}: v_{i+1}>v_{i}} \gamma^{a}\left(v_{i} v_{i+1}\right) \prod_{i \in \mathbb{Z}_{k}: v_{i+1}<v_{i}} \gamma^{a}\left(v_{i} v_{i+1}\right)^{-1} \\
& =g^{\left|\left\{i \in \mathbb{Z}_{k}: v_{i+1}>v_{i}\right\}\right|-\mid\left\{i \in \mathbb{Z}_{k}: v_{i+1}<v_{i} \mid\right\}}=g^{\delta(C)}
\end{aligned}
$$

Since $g^{\delta(C)}$ is the identity if and only if $a \mid \delta(C)$, the characterisation (iii) follows.

We also prove an easy folklore estimate for the number of (unordered) paths and circuits in a complete graph. This only serves to make the bounds in our final theorem slightly cleaner.

Lemma 2.2. $K_{n}$ has at most $2 n$ ! paths and at most $2(n-1)$ ! circuits.

Proof. Let $p_{n}, c_{n}$ denote the number of paths and circuits respectively in $K_{n}$. Using $4-e>1$, we have $p_{n}=n+\sum_{i=2}^{n}\left(\begin{array}{c}n \\ i\end{array}\right) \frac{i !}{2}<\frac{1}{2} n+\frac{e}{2} n !<2 n$ !. That $c_{n} \leq 2(n-1)$ ! is easy to verify for $n \leq 4$; suppose that $n \geq 5$. Each circuit of $K_{n}$ that is not a circuit of $K_{n-1}$ corresponds to a nonempty path of $K_{n-1}$, together with vertex $n$ added, so $c_{n} \leq p_{n-1}+c_{n-1}$ for each $n \geq 4$. Now, we inductively get $c_{n} \leq c_{n-1}+p_{n-1} \leq 2(n-2) !+\frac{e}{2}(n-1) !<$ $2(n-1)$ ! (where we use $\left.n-1>\frac{4}{4-e}\right)$, and the bound follows.

\section{Bipartite Graphs}

In this section and what follows, we use the following statement of Ramsey's theorem, as well as the very well-known bound $R_{2}(t, t) \leq 2^{2 t}$.

Theorem 3.1 (Ramsey's Theorem). There is a function $R_{k}\left(n_{1}, \ldots, n_{\ell}\right)$ so that, if $Z$ is a finite set with $R_{k}\left(n_{1}, \ldots, n_{\ell}\right)$ and each element of $\left(\begin{array}{l}Z \\ k\end{array}\right)$ is assigned a colour from $\left\{c_{1}, \ldots, c_{\ell}\right\}$, then there is some $i \in[\ell]$ and $a$ set $X \subseteq Z$ for which $|X|=k_{i}$ and each set in $\left(\begin{array}{l}X \\ k\end{array}\right)$ receives colour $c_{i}$.

Call a set of circuits $\mathcal{C}$ in a biased graph $(G, \mathcal{B})$ consistent if either $\mathcal{C} \subseteq \mathcal{B}$ or $\mathcal{C} \cap \mathcal{B}=\varnothing$. Call a biased graph $(G, \mathcal{B})$ consistent if the set of all its circuits is consistent. For a set $X \subseteq V(G)$, write $(G, \mathcal{B}) \mid X$ for the biased subgraph $\left(H, \mathcal{B}^{\prime}\right)$ where $H$ is the subgraph of $G$ induced by $X$. We now restate and prove Theorem 1.3 .

Theorem 3.2. Let $t \geq 1$ be an integer. If $n \geq 2^{2^{2^{4 t}}}$ and $(G, \mathcal{B})$ is a biased graph with $G \cong K_{n, n}$, then $(G, \mathcal{B})$ has a consistent $K_{t, t}$-subgraph. 


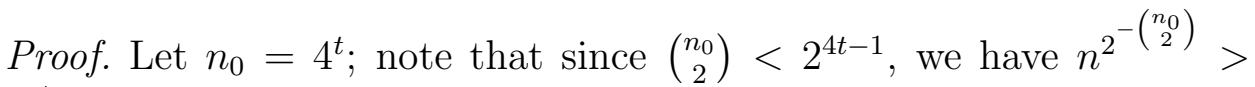
$2^{2^{t}}>t+2(2 t-1)$ !. Let $(A, B)$ be a bipartition of $G$. Let $A_{0}$ be an $n_{0^{-}}$ element subset of $A$. Let $X_{1}, X_{2}, \ldots, X_{\left(\begin{array}{c}n_{0} \\ 2\end{array}\right)}$ be an enumeration of $\left(\begin{array}{c}A_{0} \\ 2\end{array}\right)$. Let $k$ be maximal so that $0 \leq k \leq\left(\begin{array}{c}n_{0} \\ 2\end{array}\right)$ and there is a set $B_{k} \subseteq B$ with $\left|B_{k}\right| \geq n^{2^{-k}}$ such that $(G, \mathcal{B}) \mid\left(X_{i} \cup B_{k}\right)$ is consistent for each $1 \leq i \leq k$.

Suppose that $k<\left(\begin{array}{c}n_{0} \\ 2\end{array}\right)$. Let $H$ be a graph with vertex set $B_{k}$ in which vertices $u, v$ are adjacent if and only if the 4-circuit $C_{u v}$ with vertex set $\{u, v\} \cup X_{k+1}$ is balanced. Since the 4-circuits $C_{u v}, C_{u w}$ and $C_{v w}$ form a $\Theta$-graph for all distinct $u, v, w$, adjacency in $H$ is an equivalence relation, so there is a set $B_{k+1} \subseteq B_{k}$ with $\left|B_{k+1}\right| \geq \sqrt{\left|B_{k}\right|} \geq n^{2^{-(k+1)}}$ that either contains no edges of $H$, or induces a complete subgraph of $H$. Now $(G, \mathcal{B}) \mid\left(X_{i} \cup B_{k+1}\right)$ is consistent for each $1 \leq i \leq k+1$, so the existence of $B_{k+1}$ contradicts the maximality of $k$.

We may therefore assume that $k=\left(\begin{array}{c}n_{0} \\ 2\end{array}\right)$; let $B^{\prime}=B_{k}$, so $\left|B^{\prime}\right| \geq$ $n^{2^{-k}}>t+2(2 t-1)$ ! as noted earlier. Now $(G, \mathcal{B}) \mid\left(X \cup B^{\prime}\right)$ is consistent for each $X \in\left(\begin{array}{c}A_{0} \\ 2\end{array}\right)$; since $n_{0}=4^{t} \geq R_{2}(t, t)$, there is a $t$-element set $A^{\prime} \subset A_{0}$ such that the set of 4-circuits of $(G, \mathcal{B}) \mid\left(A^{\prime} \cup B^{\prime}\right)$ is consistent.

Let $B^{\prime \prime} \subseteq B^{\prime}$ be maximal so that every circuit in $G \mid\left(A^{\prime} \cup B^{\prime \prime}\right)$ is unbalanced. We may assume that $\left|B^{\prime \prime}\right|<t$, as otherwise $G \mid\left(A^{\prime} \cup B^{\prime \prime}\right)$ has a consistent $K_{t, t^{-}}$subgraph; thus $\left|A^{\prime} \cup B^{\prime \prime}\right| \leq 2 t-1$. By maximality, each $b \in B^{\prime}-B^{\prime \prime}$ is in some balanced circuit $\left[b, x_{1}, \ldots, x_{\ell}\right]$ where $x_{1}, \ldots, x_{\ell}$ is a path. There are at most $2(2 t-1) !<\left|B^{\prime}-B^{\prime \prime}\right|$ such paths, so there exist $b, b^{\prime} \in B^{\prime \prime}-B^{\prime}$ and $x_{1}, \ldots, x_{\ell} \in A^{\prime} \cup B^{\prime \prime}$ for which $\left[b, x_{1}, \ldots, x_{\ell}\right] \in \mathcal{B}$ and $\left[b^{\prime}, x_{1} \ldots, x_{\ell}\right] \in \mathcal{B}$. By the $\Theta$-property we have $\left[b, x_{1}, b^{\prime}, x_{\ell}\right] \in \mathcal{B}$; since the set of 4-circuits of $G \mid\left(A^{\prime} \cup B^{\prime}\right)$ is consistent, it follows that every 4-circuit of $G \mid\left(A^{\prime} \cup B^{\prime}\right)$ is balanced.

We now argue that every circuit of $G \mid\left(A^{\prime} \cup B^{\prime}\right)$ is balanced; since $\left|B^{\prime}\right| \geq\left|A^{\prime}\right|=t$, this implies the result. Indeed, a minimum-length unbalanced circuit $C=\left[c_{1}, \ldots, c_{2 k}\right]$ must have length greater than 4 , but then $\left[c_{1}, c_{4}, c_{5}, \ldots, c_{k}\right]$ and $\left[c_{1}, c_{2}, c_{3}, c_{4}\right]$ are smaller (and thus balanced) circuits forming a theta with $C$, a contradiction.

\section{Cliques}

We first prove a Ramsey-type result that will essentially find one of our three unavoidable examples in a large biased clique; however, recognising that the final outcome is (almost) $K_{t}^{a}$ for some $a$ will require a lemma from the next section. The proof of the following lemma uses the estimates from Lemma 2.2 . 
Lemma 4.1. Let $r, s, t \geq 1$ and $n \geq R_{4}(3 r$ !, $3 s !, 5, t)$. If $(G, \mathcal{B}, \leq)$ is an ordered biased graph with $G \cong K_{n}$, then either

- $G$ contains a $K_{r}$-subgraph in which no circuit is balanced,

- $G$ contains a $K_{s}$-subgraph in which the balanced circuits are precisely the oscillating circuits, or

- $G$ contains a $K_{t}$-subgraph in which all 1243-circuits and 1324circuits are balanced.

Proof. Suppose for a contradiction that $G$ has none of the above subgraphs. Let $\left(m_{\mathrm{b}, \mathrm{b}}, m_{\mathrm{b}, \mathrm{u}}, m_{\mathrm{u}, \mathrm{b}}, m_{\mathrm{u}, \mathrm{u}}\right)=(3 r !, 3 s !, 5, t)$. For each $Q \in\left(\begin{array}{c}V \\ 4\end{array}\right)$, let $c(Q)=(\alpha, \beta)$, where $\alpha=\mathrm{b}$ if the unique 1423-circuit on $X$ is balanced, and $\alpha=\mathrm{u}$ otherwise, and $\beta \in\{\mathrm{b}, \mathrm{u}\}$ is defined similarly for the unique 1243-circuit on $X$. By the definition of $n$, there is a subgraph $H$ of $G$ and some $\left(\alpha_{0}, \beta_{0}\right) \in\{\mathrm{b}, \mathrm{u}\}^{2}$ such that $c(Q)=\left(\alpha_{0}, \beta_{0}\right)$ for all $Q \in\left(\begin{array}{c}V(H) \\ 4\end{array}\right)$, and $|V(H)|=m_{\alpha_{0}, \beta_{0}}$. Let $U=V(H)$.

4.1.1. If $\beta_{0}=\mathrm{b}$ then $\alpha_{0}=\mathrm{b}$.

Proof. Suppose not; so $\left(\alpha_{0}, \beta_{0}\right)=(\mathrm{u}, \mathrm{b})$ and $|U|=5$; let $v_{1}, \ldots, v_{5}$ be the vertices of $U$ in increasing order. The $\Theta$-graph containing circuits $\left[v_{1}, v_{2}, v_{4}, v_{3}\right],\left[v_{1}, v_{2}, v_{5}, v_{3}\right]$ and $\left[v_{2}, v_{4}, v_{3}, v_{5}\right]$ contains two (balanced) 1243-circuits and one (unbalanced) 1324-circuit, a contradiction.

4.1.2. $\alpha_{0}=$ b.

Proof. If not, then $\left(\alpha_{0}, \beta_{0}\right)=(\mathbf{u}, \mathbf{u})$, so $|U|=3 r$ !. Let $X \subseteq U$ be maximal such that $X$ contains no balanced circuits. If $|X| \geq r$ then $G$ has a $K_{r}$-subgraph with no balanced circuits, a contradiction; thus $|X| \leq r-1$, so $U-X$ has a partition into at most $r$ intervals, and $|U-X|>3 r !-r>2 r(r-1)$ !. By maximality, for each $v \in U-X$ there is a balanced circuit $C(v)$ contained in $X \cup\{v\}$. For each such $v$, the graph $P(v)=C(v)-v$ is a path contained in $X$, and there are at most $2(r-1)$ ! such paths; by a majority argument there exist $v_{1}, v_{2} \in U-X$ for which $P\left(v_{1}\right)=P\left(v_{2}\right)$, and $v_{1}$ and $v_{2}$ lie in the same interval of $U-X$. Now $C\left(v_{1}\right)$ and $C\left(v_{2}\right)$ form a $\Theta$-subgraph with a 4-circuit $C=\left[u, v_{1}, w, v_{2}\right]$ where $u, w$ are the ends of $P\left(v_{1}\right)$, so $C$ is balanced. Since $u$ and $w$ do not lie in the interval between $v_{1}$ and $v_{2}, C$ is either a 1324-circuit or a 1243-circuit. Thus either $\alpha_{0}=\mathrm{b}$ or $\beta_{0}=\mathrm{b}$, and the claim follows from 4.1.1.

The above claim gives that all 1324-circuits in $H$ are balanced.

4.1.3. All oscillating circuits in $H$ are balanced.

Proof. Suppose otherwise, and let $C$ be an unbalanced oscillating circuit with $t$ as small as possible, with canonical ordering $v_{1}, \ldots, v_{t}$. Note 
that $v_{1}<v_{3}<\min \left(v_{2}, v_{4}\right)$ and $t$ is even. Clearly $t>4$, since all oscillating circuits of length at most 4 are 1324-circuits. The circuit $C^{\prime}=\left[v_{1}, v_{4}, v_{5} \ldots, v_{t}\right]$ is also oscillating, and is balanced by minimality. Now $C$ and $C^{\prime}$ are in a $\Theta$-graph with the (balanced) 1324-circuit $\left[v_{1}, v_{2}, v_{3}, v_{4}\right]$, contradicting the choice of $C$ as unbalanced.

4.1.4. $\beta_{0}=\mathrm{b}$.

Proof. Suppose otherwise, so $\left(\alpha_{0}, \beta_{0}\right)=(\mathrm{b}, \mathrm{u})$ (i.e. all 1243-circuits of $H$ are unbalanced) and $|U|=3 s$ !. Call a circuit bad if it is balanced but not oscillating. Let $n_{1}=2(s-1)$ !, and let $U_{0}, U_{1}, \ldots, U_{s}$ be sets of vertices of $H$ such that $\left|U_{0}\right|=s$, while for each $0<i \leq s$ we have $\left|U_{i}\right|=n_{1}$, and the vertices of $U_{i-1}$ precede the vertices of $U_{i}$ in the ordering; these sets exist because $s+s n_{1}<s+2 s !<|U|$.

Let $u_{1}, \ldots, u_{s}$ be the vertices of $U_{0}$ listed in increasing order. Let $\mathcal{C}$ denote the set of circuits of $H$ whose vertex set is contained in $U_{0}$ (noting that $|\mathcal{C}|<n_{1}$ ), and let $\mathcal{W}=U_{1} \times U_{2} \times \ldots \times U_{s}$. For each $1 \leq i \leq s$ and $W=\left(w_{1}, \ldots, w_{s}\right) \in \mathcal{W}$, let $\varphi_{W}\left(u_{s}\right)=w_{s}$. For each $C \in \mathcal{C}$, let $\varphi_{W}(C)$ denote the circuit obtained from $C$ by applying the $\operatorname{map} \varphi_{W}$ to each vertex.

Note that the map $C \mapsto \varphi_{W}(C)$ is a bijection between $\mathcal{C}$ and the set of circuits with vertex set contained in $W$, and that this map preserves the property of being oscillating.

Let $W=\left(w_{1}, \ldots, w_{s}\right) \in \mathcal{W}$ be such that the number of bad circuits with vertex set contained in $W$ is as small as possible. If this number is zero, then $W$ is the vertex set of a $K_{s}$-subgraph in which precisely the oscillating circuits are balanced, a contradiction. Thus, there is some $C_{0} \in \mathcal{C}$ for which $\varphi_{W}\left(C_{0}\right)$ is bad, so, since $C_{0}$ is not oscillating, there are consecutive vertices $x, y, z$ in $C_{0}$ with $x<y<z$. Let $A$ be the set $U_{i}$ containing $\varphi_{W}(y)$, and for each $a \in A$, let $W_{a}$ be obtained from $W$ by replacing $\varphi_{W}(y)$ with $a$. Note that if $C \in \mathcal{C}$, then either

(1) $y \notin V(C)$ and $\varphi_{W_{a}}(C)=\varphi_{W_{a^{\prime}}}(C)$ for all $a, a^{\prime} \in A$,

(2) $y$ is either the minimum or maximum of its two neighbours in $C$ and, for all $a, a^{\prime} \in A$, the circuits $\varphi_{W_{a}}(C)$ and $\varphi_{W_{a^{\prime}}}(C)$ form a $\Theta$-graph with a 1324-circuit (which is balanced by (4.1.2)), or

(3) $y$ lies between its two neighbours in $C$ and, for all $a, a^{\prime} \in A$, the circuits $\varphi_{W_{a}}(C)$ and $\varphi_{W_{a}^{\prime}}(C)$ form a $\Theta$-graph with an 1243circuit (which is unbalanced by assumption).

For each $C \in \mathcal{C}$, consider the set $A_{C}$ of elements $a \in A$ for which $\varphi_{W_{a}}(C)$ is balanced and $\varphi_{W}(C)$ is unbalanced. Note that if (1) holds then $A_{C}=\varnothing$. If (2) holds, then for each $a \in A_{C}$, the $\Theta$-graph containing some 1324-circuit and the circuits $\varphi_{W}(C)$ and $\varphi_{W_{a}}(C)$ contains 
exactly two balanced circuits, so $A_{C}=\varnothing$. If (3) holds and $a, a^{\prime} \in A_{C}$ are distinct, then the $\Theta$-graph containing $\varphi_{W_{a}}(C), \varphi_{W_{a^{\prime}}}(C)$, and some 1243-circuit contains exactly two balanced circuits, so $\left|A_{C}\right| \leq 1$.

Since $|\mathcal{C}|<n_{1}=|A|$, there is some $b \in A$ that is outside every $A_{C}$. Note that $\varphi_{W}\left(C_{0}\right)$ is balanced and that, since $y$ lies (in the ordering) between its two neighbours in $C_{0}$, the circuit $C_{0}$ satisfies (3) and, since 1243-circuits are unbalanced, so is the circuit $\varphi_{W_{b}}\left(C_{0}\right)$. So $\varphi_{W_{b}}\left(C_{0}\right)$ is not bad. Moreover, by choice of $b$, there is no $C \in \mathcal{C}$ for which $\varphi_{W}(C)$ is not bad and $\varphi_{W_{b}}(C)$ is bad. Therefore $W_{b}$ contains strictly fewer bad circuits than $W$, contradicting the choice of $W$.

By these claims, we have $\left(\alpha_{0}, \beta_{0}\right)=(\mathrm{b}, \mathrm{b})$ and $|U|=t$; thus, $H$ satisfies the third outcome, a contradiction.

\section{BALANCED 4-CIRCUITS}

Let $(G, \leq)$ be an ordered graph where $G$ is complete with vertex set $V$. Define $\Omega(G)$ to be the graph whose vertices are the nonspanning circuits of $G$, in which two circuits $C_{1}, C_{2}$ are adjacent if and only if there is a $\Theta$-subgraph of $G$ containing $C_{1}, C_{2}$ and some 1243-circuit or 1324-circuit. Our first lemma describes the components of $\Omega$.

Lemma 5.1. Let $n \geq 5$ and let $(G, \leq)$ be an ordered graph where $G \cong K_{n}$. Then two vertices $F_{1}, F_{2}$ of $\Omega(G)$ are in the same component of $\Omega$ if and only if $\delta\left(F_{1}\right)=\delta\left(F_{2}\right)$.

Proof. We may assume that $G=K_{n}$ and $\leq$ is the usual ordering. Let $\Omega=\Omega(G)$. Say that two vertices of $\Omega$ are connected if they are in the same component of $\Omega$.

5.1.1. Each pair of similar circuits of $G$ is connected in $\Omega$.

Subproof: It suffices to show for each $3 \leq k \leq n$ that each $k$-circuit of $G$ is connected in $\Omega$ to the unique circuit with vertex set $[k]$ that is similar to $C$. Let $C_{0}$ be a $k$-circuit such that $C, C_{0}$ are connected in $\Omega$, while the sum $\Sigma\left(C_{0}\right)$ of the vertices in $C_{0}$ is as small as possible. If $V\left(C_{0}\right)=[k]$ then the required statement holds. Otherwise there is some $\ell \in V\left(C_{0}\right)$ for which $\ell-1 \notin V\left(C_{0}\right)$. Let $C_{0}^{\prime}$ be obtained by replacing the vertex $\ell$ with the vertex $\ell-1$ in $C^{\prime}$. Now $C_{0}^{\prime}$ and $C_{0}$ form a $\Theta$-graph with a 4 -circuit $C^{\prime \prime}=[x, \ell-1, y, \ell]$; since no vertex lies between $\ell-1$ and $\ell$ in the ordering, we see that $C_{4}$ is a 1243 - or 1324-circuit. Thus $X_{0}^{\prime}$ and $C_{0}^{\prime}$ are adjacent in $\Omega$; since $\Sigma C_{0}^{\prime}=\Sigma C_{0}-1$, this contradicts the minimality in the choice of $C_{0}$. 
Call a circuit $C$ of $G$ basic if either $C=[1,2,4,3]$, or if $C=[1, \ldots, k]$ for some $k \geq 3$. Note that the first type has $\delta=0$, while other basic $k$-circuits have $\delta=k-2$.

5.1.2. Each nonspanning circuit of $G$ is connected in $\Omega$ to a basic circuit.

Subproof: For each circuit $C$ of $G$ with canonical ordering $v_{1}, \ldots, v_{k}$, call a pair $(i, j)$ decreasing in $C$ if $1 \leq i<j \leq k$ and $v_{i}>v_{j}$; since the ordering is canonical, any decreasing pair $(i, j)$ satisfies $i>1$. Similar circuits have the same set of decreasing pairs.

Fix a circuit $C$ of $G$. Let $C^{\prime}$ be a circuit connected to $C$ in $\Omega$ for which $\left|V\left(C^{\prime}\right)\right|$ is as small as possible, and, subject to this, $C^{\prime}$ has as few decreasing pairs as possible. Let $C_{0}$ be the unique circuit with vertex set $[k]$ that is similar to $C^{\prime}$; we show that $C_{0}$ is basic. Let $v_{1}, v_{2}, \ldots, v_{k}$ be the canonical ordering of $C_{0}$, so $v_{1}=1$ and $v_{2}<v_{k}$.

Note that $C$ is connected to $C_{0}$ in $\Omega$, and that the decreasing pairs for $C_{0}$ are exactly those for $C^{\prime}$. If $C_{0}$ has no decreasing pair then $C_{0}=$ $[1, \ldots, k]$ is basic, giving the result. If $k \leq 4$ then $C_{0}$ is either one of the basic circuits $[1,2,3],[1,2,3,4],[1,2,4,3]$, or $C_{0}=[1,3,2,4]$. In the first three cases the result holds, and in the last, the $\Theta$-graph containing circuits $C_{0},[3,5,2,4]$ and $[1,3,5,4]$ certifies that $C_{0}$ and $[1,3,5,4]$ are adjacent in $\Omega$, since $[1,3,5,4]$ is similar to $[1,2,4,3]$, the result holds by (5.1.1).

Suppose, therefore, that $k>4$ and that $C_{0}$ has a decreasing pair. Let $(a, b)$ be a decreasing pair for $C_{0}$ for which $a$ is as small as possible, and subject to this, $b$ is as large as possible. Note that $a>1$. Suppose first that $b=a+1$; since $(a-1, a+1)$ is not decreasing we have $v_{a-1}<v_{a+1}<v_{a}$.

If $a=2$ then, since $(2,4)$ is not decreasing we have $v_{1}<v_{3}<v_{2}<v_{4}$. Let $C_{1}=\left[v_{1}, v_{4}, v_{5} \ldots, v_{k}\right]$; note that $\left|V\left(C_{1}\right)\right|=k-2 \geq 3$. Now $C_{0}$ and $C_{1}$ form a $\Theta$-graph with the 1324 -circuit $C_{2}=\left[v_{1}, v_{2}, v_{3}, v_{4}\right]$. Therefore $C_{1}$ is adjacent to $C_{0}$ in $\Omega$ and is thus connected to $C$; since $\left|V\left(C_{1}\right)\right|<\left|V\left(C^{\prime}\right)\right|$, this contradicts the minimality in the choice of $C^{\prime}$.

If $a>2$ then since $(a-2, a-1)$ is not decreasing we have have $v_{a-2}<v_{a-1}<v_{a+1}<v_{a}$. Let $C_{1}=\left[v_{1}, \ldots, v_{a-2}, v_{a+1}, \ldots, v_{k}\right]$ (noting that $\left.\left|V\left(C_{1}\right)\right|=k-2 \geq 3\right)$. The circuits $C_{0}$ and $C_{1}$ form a $\Theta$-graph with the 1243-circuit $C_{2}=\left[v_{a-2}, v_{a-1}, v_{a}, v_{a+1}\right]$; we obtain a contradiction as in the previous case.

We may thus assume that $b>a+1$. We will now derive a contradiction by finding a $k$-circuit $C_{0}^{\prime \prime}$, connected to $C$ in $\Omega$, with fewer decreasing pairs than $C$. 
Let $C_{0}^{\prime}=\left[v_{1}^{\prime}, v_{2}^{\prime}, \ldots, v_{k}^{\prime}\right]$ where $v_{i}^{\prime}=v_{i}$ if $v_{i}<v_{b}$, and $v_{i}^{\prime}=v_{b}+1$ if $v_{i} \geq v_{b}$. So $C_{0}^{\prime}$ has vertex set $[k+1]-\left\{v_{b}\right\}$ and is similar to $C_{0}$ (and the ordering $v_{1}^{\prime}, \ldots, v_{k}^{\prime}$ is canonical). Let $C_{0}^{\prime \prime}=\left[v_{1}^{\prime \prime}, \ldots, v_{k}^{\prime \prime}\right]$, where $v_{i}^{\prime \prime}=v_{i}^{\prime}$ if $i \neq a$, and $v_{a}^{\prime \prime}=v_{b}$. Again, since $v_{2}^{\prime \prime} \leq v_{2}^{\prime}<v_{k}^{\prime}=v_{k}^{\prime \prime}$, the ordering $v_{1}^{\prime \prime}, \ldots, v_{k}^{\prime \prime}$ is canonical. The circuits $C_{0}^{\prime \prime}$ and $C_{0}^{\prime}$ form a $\Theta$-graph with the 4-circuit $C_{1}=\left[v_{a-1}^{\prime}, v_{b}, v_{a+1}^{\prime}, v_{a}^{\prime}\right]$. Since $v_{b}^{\prime}=v_{b}+1 \notin V\left(C_{1}\right)$, the circuit $C_{1}$ is similar to $C_{1}^{\prime}=\left[v_{a-1}^{\prime}, v_{b}^{\prime}, v_{a+1}^{\prime}, v_{a}^{\prime}\right]$; the fact that $v_{b}^{\prime}<v_{a}^{\prime}$ and neither $(a-1, b)$ nor $(a, a+1)$ is decreasing in $C_{0}^{\prime}$ gives $v_{a-1}^{\prime}<v_{b}^{\prime}<v_{a}^{\prime}<v_{a+1}^{\prime}$ and so $C_{1}^{\prime}$ and $C_{1}$ are 1243 -circuits. This implies that $C_{0}^{\prime}$ and $C_{0}^{\prime \prime}$ are adjacent in $\Omega$.

Since $v_{a}^{\prime \prime}=v_{b}<v_{b}+1=v_{b}^{\prime \prime}$, the pair $(a, b)$ is not decreasing in $C_{0}^{\prime \prime}$ but is decreasing in $C_{0}$ and therefore in $C^{\prime}$. We now argue that every decreasing pair $(i, j)$ in $C_{0}^{\prime \prime}$ is decreasing in $C_{0}^{\prime}$; this will imply that $C_{0}^{\prime \prime}$ has strictly fewer decreasing pairs than $C_{0}$ and therefore fewer such pairs than $C^{\prime}$, contradicting the minimality in the choice of $C^{\prime}$.

Let $(i, j)$ be a decreasing pair in $C_{0}^{\prime \prime}$. If $j=a$ then $i<a$ and, using the fact that $(a-1, b)$ is not decreasing in $C_{0}^{\prime}$, we have $v_{i}^{\prime \prime}=v_{i}^{\prime} \leq v_{a-1}^{\prime}<$ $v_{b}^{\prime}=v_{b}+1=v_{a}^{\prime \prime}+1$ and so $v_{i}^{\prime \prime} \leq v_{a}^{\prime \prime}=v_{j}^{\prime \prime}$, contradicting the definition of $(i, j)$. If $j \neq a$ then, using the fact that $v_{\ell}^{\prime} \geq v_{\ell}^{\prime \prime}$ for all $\ell$, we have $v_{i}^{\prime} \geq v_{i}^{\prime \prime}>v_{j}^{\prime \prime}=v_{j}^{\prime}$ so $(i, j)$ is decreasing in $C_{0}^{\prime}$. This fact establishes the aforementioned contradiction.

5.1.3. If $C_{1}, C_{2}$ are connected in $\Omega$, then $\delta\left(C_{1}\right)=\delta\left(C_{2}\right)$.

Subproof: It suffices to show this for $C_{1}, C_{2}$ adjacent in $\Omega$. Let $H$ be a $\Theta$-graph of $\Omega$ containing $C_{1}, C_{2}$ and a 1324-circuit or 1243-circuit $C_{3}$; note that $\delta\left(C_{3}\right)=0$. Let $x, y$ be the vertices of $H$ for which $E(H)$ is the disjoint union of three $x y$-paths $P_{1}, P_{2}, P_{3}$, where each $C_{i}$ has edge set disjoint from that of $P_{i}$. For each $x y$-path $P$ with vertices $x=x_{0}, x_{1}, \ldots, x_{\ell}=y$ listed in path order, let

$$
\delta(P)=\left|\left\{i \in[\ell]: x_{i}>x_{i-1}\right\}\right|-\left|\left\{i \in[\ell]: x_{i}<x_{i-1}\right\}\right| .
$$

By construction, we have $\left(\delta\left(C_{1}\right), \delta\left(C_{2}\right), \delta\left(C_{3}\right)\right)=\left(\left|\delta\left(P_{3}\right)-\delta\left(P_{2}\right)\right|,\left|\delta\left(P_{1}\right)-\delta\left(P_{3}\right)\right|,\left|\delta\left(P_{2}\right)-\delta\left(P_{1}\right)\right|\right)$. Since $\delta\left(C_{3}\right)=0$, this gives $\delta\left(P_{1}\right)=\delta\left(P_{2}\right)$ and so $\delta\left(C_{1}\right)=\delta\left(C_{2}\right)$.

By (5.1.3), vertices of $\Omega$ in the same component have the same value of $\delta$. By (5.1.2), any two vertices $C_{1}, C_{2}$ of $\Omega$ with $\delta\left(C_{1}\right)=\delta\left(C_{2}\right)=d$ are both connected to the unique basic circuit $C_{0}$ with $\delta\left(C_{0}\right)=d$, so are connected in $\Omega$; the lemma follows.

Lemma 5.2. Let $n \geq 5$ and let $(G, \mathcal{B}, \leq)$ be an ordered biased graph with $G \cong K_{n}$. If all 1324-circuits and 1243-circuits of $G$ are balanced, then there exists $a>0$ such that $(G, \mathcal{B})-v \cong K_{n-1}^{a}$ for each $v \in V$. 
Proof. We may assume that $(G, \leq)$ is $K_{n}$ with the usual ordering. Let $\Omega=\Omega(G)$. Note that a nonspanning circuit $C$ of $G$ must have $\delta(C) \leq$ $|V(C)|-2 \leq n-3$. For each $0 \leq d \leq n-3$, let $\mathcal{U}_{d}$ be the set of nonspanning circuits $C$ of $G$ with $\delta(C)=d$. Note that each $\mathcal{U}_{d}$ is nonempty; by Lemma 5.1, the $\mathcal{U}_{d}$ are the components of $\Omega$.

By the definition of $\Omega$, hypothesis, and the $\Theta$-property, adjacent vertices of $\Omega$ are either both balanced or both unbalanced; it follows that for each $d \geq 0$, we either have $\mathcal{U}_{d} \subseteq \mathcal{B}$ or $\mathcal{U}_{d} \cap \mathcal{B}=\varnothing$. Let $\mathcal{D}$ be the set of all $0 \leq d \leq n-3$ for which $\overline{\mathcal{U}}_{d} \subseteq \mathcal{B}$. Since $\mathcal{U}_{0} \cap \mathcal{B}$ contains the circuit $[1,2,4,3]$, we have $0 \in \mathcal{D}$.

Let $d_{1}, d_{2} \in \mathcal{D}$ with $0<d_{1}<d_{2}$; we show that $d_{2}-d_{1} \in \mathcal{D}$. Let $H$ be the $\Theta$-graph containing $C_{2}=\left[1,2, \ldots, d_{2}+2\right]$ together with a single edge from 1 to $d_{1}+2$. We have $\delta\left(C_{2}\right)=d_{2}$, and the other two circuits $C_{0}, C_{1}$ of $H$ satisfy $\delta\left(C_{0}\right)=d_{2}-d_{1}$ and $\delta\left(C_{1}\right)=d_{1}$. Since $d_{1}, d_{2} \in \mathcal{D}$ we have $\left\{C_{1}, C_{2}\right\} \subseteq \mathcal{B}$ and so $C_{0} \subseteq \mathcal{B} \cap \mathcal{U}_{d_{2}-d_{1}}$ by the $\Theta$ property. This implies that $\mathcal{U}_{d_{2}-d_{1}} \subseteq \mathcal{B}$ and so $d_{2}-d_{1} \subseteq \mathcal{D}$. Thus $\mathcal{D}$ contains zero and is closed under absolute differences. It follows that there exists $a>0$ for which $\mathcal{D}=\{d \in\{0, \ldots, n-3\}: a \mid d\}$, and thus a nonspanning circuit $C$ of $G$ is balanced if and only if $a \mid \delta(C)$. Thus $(G, \mathcal{B})-v \cong K^{a}(n-1)$ for each vertex $v$ of $G$.

Combining this Lemma with Theorem 4.1 and Lemma 2.1, we get the following restatement of our main theorem with explicit bounds in terms of Ramsey numbers and our stronger, 'ordered' isomorphism.

Theorem 5.3. Let $r, s \geq 1, t \geq 4$ and $n \geq R_{4}(3 r$ !, $3 s !, 5, t+1)$. If $(G, \mathcal{B}, \leq)$ is an ordered biased graph with $G \cong K_{n}$, then $(G, \mathcal{B}, \leq)$ has an ordered biased subgraph isomorphic to $K_{r}^{\mathbf{u}}, K_{s}^{\mathbf{o}}$, or $K_{t}^{a}$ for some $a>0$.

Finally, we give a construction implying that the vertex deletion in Lemma 5.2 is necessary, in fact showing that there are doubly exponentially many counterexamples if the vertex deletion is not insisted upon. Since Lemma 5.2 controls the behaviour of every nonspanning cycle, all the wildness occurs within the Hamilton cycles.

Theorem 5.4. For all $n \geq 10$, there are at least $2^{2^{n-9}}$ ordered biased graphs $(G, \mathcal{B}, \leq)$ where $G=K_{n}$ and every 1243 -circuit and 1324 -circuit of $G$ is balanced, but $(G, \mathcal{B})$ does not arise from any group-labelling.

Proof. Let $\mathcal{P}$ be the collection of ordered partitions $(I, J)$ of $[n]$ for which $\{1,2, n-2, n-1\} \subseteq I$ and $\{3,4,5, n\} \subseteq J$, so $|\mathcal{P}|=2^{n-8}$. Let $G=(V, E)=K_{n}$ and let $(G, \mathcal{B}, \leq)=K^{a}(n)$ where $a=n-4$. For each $(I, J) \in \mathcal{P}$, let $C_{I, J}$ be the $n$-circuit with canonical ordering 
$u_{1}, \ldots, u_{\ell}, v_{1}, \ldots, v_{k}$, where the $u_{i}$ are the elements of $I$ in increasing order and the $v_{j}$ are the elements of $J$ in increasing order. Note that $\delta\left(C_{I, J}\right)=n-4$ and therefore that $C_{I, J} \in \mathcal{B}^{\prime}$ for each $(I, J)$, and that the map $(I, J) \mapsto C_{I, J}$ is injective. For each $\mathcal{Q} \subseteq \mathcal{P}$, let $\mathcal{B}_{\mathcal{Q}}=$ $\mathcal{B}-\left\{C_{I, J}:(I, J) \in \mathcal{Q}\right\}$.

5.4.1. $\left(G, \mathcal{B}_{\mathcal{Q}}\right)$ is a biased graph for all $\mathcal{Q} \subseteq \mathcal{P}$.

Subproof: If not, then there is a $\Theta$-subgraph $H$ containing exactly two balanced circuits. No such subgraph exists in $(G, \mathcal{B})$, so it must be the case that $H$ contains an unbalanced $n$-circuit $C_{0}=C_{I, J}$ for some $(I, J) \in \mathcal{Q}$ and two other balanced circuits $C_{1}, C_{2}$; since no $\Theta$-subgraph of $K_{n}$ contains two $n$-circuits, we must have $C_{1}, C_{2} \in \mathcal{B}$. Let $P_{0}, P_{1}, P_{2}$ be the three $x y$-paths so that $E\left(C_{i}\right)=E(H)-E\left(P_{i}\right)$ for each $i \in$ $\{0,1,2\}$. Since $C_{0}$ is an $n$-circuit, the path $P_{0}$ is a chord of $C_{0}$, so $\left|E\left(P_{0}\right)\right|=1$ and $\left|E\left(P_{1}\right)\right|+\left|E\left(P_{2}\right)\right|=n$. As in the proof of (5.1.3), let

$$
\delta(P)=\left|\left\{i \in[\ell]: x_{i}>x_{i-1}\right\}\right|-\left|\left\{i \in[\ell]: x_{i}<x_{i-1}\right\}\right|
$$

for each path $P$ with vertices occuring in order $x_{i}: i \in[\ell]$; so

$\left(\delta\left(C_{0}\right), \delta\left(C_{1}\right), \delta\left(C_{2}\right)\right)=\left(\left|\delta\left(P_{2}\right)-\delta\left(P_{1}\right)\right|,\left|\delta\left(P_{1}\right)-\delta\left(P_{0}\right)\right|,\left|\delta\left(P_{0}\right)-\delta\left(P_{2}\right)\right|\right)$.

We have $\delta\left(C_{0}\right)=n-4$ and, since $C_{1}, C_{2} \in \mathcal{B}$, both $\delta\left(C_{1}\right)$ and $\delta\left(C_{2}\right)$ are multiples of $n-4$. The above also gives $n-4=\delta\left(C_{0}\right)= \pm \delta\left(C_{1}\right) \pm \delta\left(C_{2}\right)$. Moreover, since $\delta\left(C_{i}\right) \leq\left|V\left(C_{i}\right)\right|-2 \leq n-3$ for each $i \in\{1,2\}$, we must have $\left\{\delta\left(C_{1}\right), \delta\left(C_{2}\right)\right\}=\{0, n-4\}$; we may assume that $\delta\left(C_{1}\right)=0$ (so $\left.\delta\left(P_{2}\right)=\delta\left(P_{0}\right)\right)$ and $\delta\left(C_{2}\right)=n-4$. Thus $n-4=\delta\left(C_{2}\right) \leq\left|V\left(C_{2}\right)\right|-2$ and so $\left|V\left(C_{2}\right)\right| \geq n-2$; since $\delta\left(C_{2}\right) \equiv\left|V\left(C_{2}\right)\right|(\bmod 2)$ and $\left|V\left(C_{2}\right)\right|<n$ we must have $\left|V\left(C_{2}\right)\right|=n-2$. Thus $\left|E\left(P_{2}\right)\right|=|E(H)|-\left|E\left(C_{2}\right)\right|=$ $(n+1)-(n-2)=3$ and so $C_{1}$ is a 4 -cycle with $\delta\left(C_{1}\right)=0$.

However, the construction of $\mathcal{P}$ and $C_{0}=C_{I, J}$ gives that $C_{0}=$ $\left[u_{1}, u_{2}, \ldots, u_{\ell}, v_{1}, v_{2}, \ldots, v_{k}\right]$ where the $u_{i}$ occur in increasing order with $\left(u_{1}, u_{2}, u_{\ell-1}, u_{\ell}\right)=(1,2, n-2, n-1)$ and the $v_{i}$ occur in increasing order with $\left(v_{1}, v_{2}, v_{3}, v_{k}\right)=(3,4,5, n)$. The 4 -cycle $C_{1}$ satisfies $C_{1}=\left[x_{1}, x_{2}, x_{3}, x_{4}\right]$ where $x_{1}, x_{2}, x_{3}, x_{4}$ are consecutive vertices in $C$. Any such quadruple of consecutive vertices is either a subsequence of $\left(u_{1}, \ldots, u_{\ell}\right)$ or $\left(v_{1}, \ldots, v_{k}\right)$, or is one of the quadruples $(n-2, n-1,3,4),(n-1,3,4,5),\left(v_{k-1}, n, 1,2\right)$ or $\left(n, 1,2, u_{3}\right)$. All these possibilities imply that $\delta\left(C_{1}\right)=2$, a contradiction.

We now prove that if $\varnothing \neq \mathcal{Q} \neq \mathcal{P}$, then $\left(G, \mathcal{B}_{\mathcal{Q}}\right)$ does not arise from a group-labelling. Since there are $2^{|\mathcal{P}|}-2>2^{2^{n-9}}$ possible $\mathcal{Q}$, this will give the lemma. Suppose it does; let $\Gamma$ be a group with identity id, and let $\gamma: E \rightarrow \Gamma$ be a labelling of the edges of some orientation $\vec{G}$ of $G$ whose balanced circuits are precisely those in $\mathcal{B}_{\mathcal{Q}}$. It is well-known and 
routine to show that applying either of the following two operations to a $\Gamma$-labelling of an orientation of a graph do not change the associated biased graph:

(a) reversing the orientation of an edge, and replacing the label of that edge with its inverse in $\Gamma$;

(b) for a vertex $v$ and a constant $\alpha \in \Gamma$, right-multiplying the label of every edge oriented towards $v$ by $\alpha$, then left-multiplying the label of every edge oriented away from $v$ by $\alpha^{-1}$.

By possibly applying (国) repeatedly, we may assume that every edge $i j$ with $i<j$ is oriented towards $j$. By then applying (b) with $\alpha=\gamma(1 v)^{-1}$ to each vertex $v \in\{2, \ldots, n\}$, we may assume that $\gamma(1 i)=\mathrm{id}$ for each $i>1$. Let $1<p<q<r \leq n$. Then the 1243 -circuit $C=[1, p, r, q]$ is balanced and so id $=\gamma(1 p) \gamma(p r) \gamma(q r)^{-1} \gamma(1 q)^{-1}=\gamma(p r) \gamma(q r)^{-1}$, giving $\gamma(p r)=\gamma(q r)$. Similarly, the 1324 -circuit $C^{\prime}=[1, q, p, r]$ is balanced, so id $=\gamma(1 q) \gamma(p q)^{-1} \gamma(p r) \gamma(r 1)^{-1}$, giving $\gamma(p q)=\gamma(p r)=\gamma(q r)$. It follows that every pair of incident edges of $G-\{1\}$ are assigned the same label by $\Gamma$; connectedness gives that all edges of $G-1$ are assigned the same label $g \in \Gamma$. Let $\gamma_{0}$ by the labelling obtained from $\gamma$ by applying (b) with $v=1$ and $\alpha=g$; now $\gamma_{0}$ assigns a label of $g$ to every edge of $G$, and $\mathcal{B}_{\mathcal{Q}}$ is the set of balanced cycles of $\left(\gamma^{\prime}, \vec{G}\right)$ by construction. But $\gamma_{0}$ is also a labelling of edges of $G$ with elements of the cyclic subgroup $\Gamma_{0}$ of $\Gamma$ generated by $g$. By the definition of $K^{a}(n)$ we therefore have $\left(G, \mathcal{B}_{\mathcal{Q}}, \leq\right)=K^{a}(n)$, where $a=0$ if $\Gamma_{0}$ is infinite, and $a=\left|\Gamma_{0}\right|$ otherwise. This implies that a circuit $C$ of $G$ is in $\mathcal{B}_{\mathcal{Q}}$ if and only if $a \mid \delta(C)$. However, if $(I, J) \in \mathcal{Q}$ and $\left(I^{\prime}, J^{\prime}\right) \in \mathcal{P}-\mathcal{Q}$, we have $C_{I, J} \notin \mathcal{B}_{Q}$ and $C_{I^{\prime}, J^{\prime}} \in \mathcal{B}_{\mathcal{Q}}$ while $\delta\left(C_{I, J}\right)=\delta\left(C_{I^{\prime}, J^{\prime}}\right)=n-4$, a contradiction.

\section{REFERENCES}

[1] M. Devos, D. Funk and I. Pivotto, When does a biased graph come from a group labelling?, Adv. Appl. Math. 61 (2014), $1-18$.

[2] T. Zaslavsky, Biased graphs. I. Bias, balance, and gains, J. Combin. Theory Ser. B 47 (1989), 32-52.

Department of Combinatorics and Optimization, University of Waterloo, Waterloo, Canada 